\section{Recuperación post anestesia: una perspectiva de los pacientes}

\section{Post-anesthesia recovery. A perspective of patients}

\section{Sr. Editor:}

El concepto de medicina perioperatoria engloba el cuidado del paciente en al pre, intra y post operatorio, donde el anestesiólogo, el cirujano y otros especialistas trabajan en equipo. El conocimiento médico propio de este campo incluye el riesgo quirúrgico, así como sus complicaciones y eventos no esperados, el manejo de los riesgos inherentes a los pacientes y el cuidado clínico en este período. La recuperación post anestesia inmediata es un hito fundamental del proceso quirúrgico, ya que una proporción importante de las complicaciones ocurren en este tiempo post operatorio $^{1,2}$, resaltando su relevancia para el logro del resultado óptimo planificado. Actualmente, las complicaciones más graves son fáciles de detectar y tratar debido al uso de monitores que permiten la detección precoz de ellas y a un buen entrenamiento del personal multidisciplinario responsable del cuidado del paciente. Sin embargo, aquellos aspectos no técnicos que podrían considerarse propios de la calidad del servicio entregado son los que están empezando a tomar gran importancia, dado que están ligados a la percepción subjetiva de los pacientes en su recuperación post operatoria. Estos factores son los más temidos por los pacientes ${ }^{3} \mathrm{y}$ actualmente marcan la diferencia. Probablemente sean los predictores de la satisfacción y de la evaluación global que los pacientes hagan de su experiencia hospitalaria.

Con el fin de conocer la opinión de nuestros pacientes en relación a estos aspectos, se realizó un análisis interno de los pacientes operados en los pabellones de cirugía general, cirugía cardiovascular, obstetricia y ginecología del Hospital Clínico de la Pontificia Universidad Católica de Chile, durante los meses de mayo y junio de 2010. Evaluamos a 342 pacientes mayores de 18 años, en su período post anestésico inmediato. El objetivo fue detectar los factores más relevantes para los pacientes, de la calidad de esta importante fase post operatoria. El análisis estadístico se realizó con el software SPSS y la comparación de las frecuencias (porcentajes) se hizo con la prueba de $\chi^{2}$. Se con- sideraron significativos todos aquellos valores de p menores a 0,05 .

De los 342 pacientes analizados, $63 \%$ fueron mujeres y $37 \%$ hombres, con una distribución etárea de $80 \%$ entre los 26 y 60 años. Tres cuartos de los procedimientos realizados fueron electivos, recibiendo anestesia general $71 \%$ de los casos.

Lo más destacado de las respuestas fueron los síntomas post operatorios, donde los principales fueron: dolor, con $49 \%$ de incidencia en algún nivel de intensidad, siendo éste el síntoma que con mayor frecuencia fue relatado por los pacientes. Le siguieron "sed" (36\%), "frío" (26\%) y "vómitos" (9\%).Al realizar un análisis de subgrupo por sexo, pudimos comprobar que las mujeres presentaron más sensación de frío y sed que los hombres ( $\mathrm{p}=0,03$ y 0,026 respectivamente), incluso al agruparlas de acuerdo a intensidad del síntoma $(\mathrm{p}=0,041)$. Sobre el dolor, $75 \%$ de los pacientes tuvieron un dolor leve, caracterizado por un valor de 4 o menos en una escala de 0 a 10 (tolerable).

Esta información es muy valiosa para el cirujano, el anestesiólogo y más aún para la institución, porque permite orientar los resultados clínicos hacia una atención de calidad efectiva, alineados tanto a la eficiencia como a la seguridad de la atención. Actualmente, en Estados Unidos de Norteamérica está funcionando el Servicio de Reportes de Calidad para Médicos (The Physician Quality Reporting $(P Q R))$, que nació como producto de una legislación que unía el pago de incentivos monetarios a los médicos de acuerdo a resultados clínicos definidos para cada institución. Por ejemplo, se evalúa el porcentaje de pacientes con cirugías de duración mayor a 60 minutos (excepto by pass cardio pulmonar) que reciben algún método de calentamiento activo, o bien que la temperatura corporal de los pacientes sea igual o superior a $36^{\circ} \mathrm{C}, 30 \mathrm{~min}$ antes y $15 \mathrm{~min}$ después del fin de la anestesia. Si cumplen sobre un valor umbral, se paga el incentivo monetario a los médicos ${ }^{4}$.

Hoy, lo que era un incentivo está amenazando con ser una medida obligatoria, cuyo cumplimento determina el pago o no del arancel de la prestación al médico, lo que se ha llamado "pago por desempeño" (pay-for-performance $)^{5}$. Es posible que esta iniciativa de gestión en la salud trascienda fronteras y llegue más temprano que tarde a nuestro país. Es en este sentido que nos estamos adelantando y actuando proactivamente, 
intentando hacer un diagnóstico de situación, de manera de dirigir los esfuerzos hacia la mejoría de aquellos indicadores que en el futuro pudieran determinar nuestra forma de trabajo, así como ser los elementos diferenciadores de la calidad de atención entre distintas instituciones.

\section{Hector J. Lacassie ${ }^{1}$, Constanza Ferdinand ${ }^{2, a}$, Hernán R. Muñoz ${ }^{1}$, Soledad Díaz ${ }^{3, a}$, Alejandra Ormazábal ${ }^{4, b}$ ${ }^{1}$ División de Anestesiología, ${ }^{2}$ Dirección de Calidad y Seguridad Asistencial, Red Salud UC, ${ }^{3}$ Servicio de Recuperación, Hospital Clínico, ${ }^{4}$ Gerencia de Estudios y Proyectos. Facultad de Medicina, Pontificia Universidad Católica de Chile. Santiago de Chile. ${ }^{a}$ Enfermera Universitaria ${ }^{b}$ Estadístico}

\section{Referencias}

1. Bothner U, Georgieff M, Schwilk B. The impact of minor perioperative anesthesia-related incidents, events, and complications on postanesthesia care unit utilization. Anesth Analg 1999; 89: 506-13.

2. Hines R, Barash PG, Watrous G, O'Connor T. Complications occurring in the postanesthesia care unit: a survey. Anesth Analg 1992; 74: 503-9.

3. Macario A, Weinger M, Carney S, Kim A. Which clinical anesthesia outcomes are important to avoid? The perspective of patients. Anesth Analg 1999; 89: 652-8.

4. Services. CFMAM. 2011 PQRS Measures List. Disponible en: http://tinyurl.com/3pfbsr9 [Consultado el 20 de mayo de 2011].

5. Woolnough M, Allam J, Hemingway C, Cox M, Yentis SM. Intraoperative Fluid Warming in Elective Cesarean Section: A Blinded Randomized Controlled Trial. Obstetric Anesthesia Digest 2010; 30: 157-9.

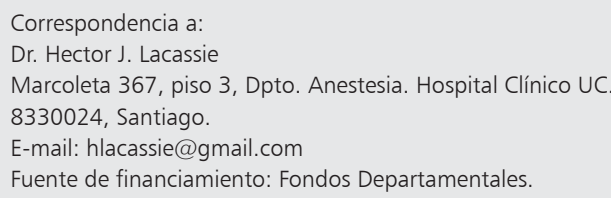

